UDC 547.789

\title{
N-ALKOXYMETHYL DERIVATIVES OF 2-AMINOTHIAZOLE AND THEIR PROTECTIVE PROPERTIES
}

\author{
V.M. Farzaliyev, M.T. Abbasova, B.G. Babaeva, M.A. Mirzoeva, G.M. Kulieva, \\ L.R. Safarova, N.A. Alieva
}

Acad. A. Guliyev Institute of Chemistry of Additives

National Academy of Sciences of Azerbaijan

Boyukshor highway, quarter 2062, Baku AZ 1029, Azerbaijan

e-mail: leyla.aki@mail.ru

Received 01.10.2020

Accepted 07.12.2020

\begin{abstract}
Conditions for alkoxymethylation of 2-aminothiazole with semiformals obtained by the interaction of aliphatic alcohols with formaldehyde were worked out. Alkoxymethylation was performed by means of preliminary preparation of the semiformal of appropriate hydroxylcontaining compounds followed by interaction of semiformal with equimolar amount of 2aminothiazole. The structure of the obtained compounds was acknowledged by means of IR and $N M R{ }^{1} H$ spectroscopy. It was established that the reaction of the alkoxymethylation proceeds in the amino form of the starting 2-aminothiazole to form an appropriate monosubstituted $\mathrm{N}$ alkoxymethyl derivative. As a result of microbiological tests, it was established that these compounds have high bactericidal properties against microorganisms which affect petroleum products and, at a concentration of 0.5\%, completely protect both M-8 lubricating oil and diesel fuel from damage by bacteria even in terms of their forced infection. It found that at a concentration of $100 \mathrm{mg} / \mathrm{l}$ the obtained compounds protect the CT-3 steel from acid corrosion, moreover $N$-(isopropoxymethyl)thiazol-2-amine has the greatest protection effect.

Keywords: 2-aminothiazole, the tautomerism, imino form, amino form, the alkoxymethylation, semiformals, alkoxymethyl derivatives, antimicrobial activity, anticorrosion properties, oil products, microorganisms, biodamage.
\end{abstract}

DOI: $10.32737 / 2221-8688-2020-4-427-435$

\section{Introducton}

As it is known that 2-aminothiazole is a potentially tautomeric compound with several<smiles>Nc1nccs1</smiles><smiles>CC1(c2ccccc2)CC1</smiles>

Studies of the effect of the type and position of the substituent on the conformational structure and amino-imine tautomerism, carried out in phases of gas, water and carbon tetrachloride showed that in these phases the amino form is more stable than the imino form and the amino tautomer is the predominant reaction centers and can enter into chemical reactions in amino and imino forms [1]:

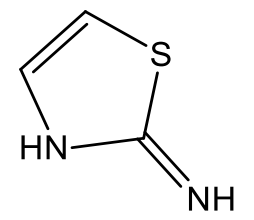


antioxidant, anti-wear properties, as well as potential antimicrobial activity.

It should be noted that 2-aminothiazole derivatives are widely used in medicine as antioxydants [3], chemotherapeutic agents [4], inhibitors of enzymes, including carboesterase [5] which are potentially powerful antidiabetic and antibacterial agents [6] demonstrating antituberculosis activity [7] and exhibiting high antimicrobial properties [8-10]. Triazine derivatives containing 2-aminothiazole fragments as a substituent are recommended as effective polyfunctional (namely anticorrosive, antiwear, and extreme pressure) additives to lubricating oils [11]. Anticorrosive and antimicrobial properties of 2-aminothiazole derivatives replaced in the position 4 by methyl, phenyl and naphthyl groups in watered jet fuel were studied $[12,13]$.

Object of these studies is to synthesize new derivatives of 2-aminothiazole and explore their protective (antimicrobial and anticorrosive) properties.

\section{Experimental part}

The presence of amine group with two active hydrogen atoms suggested the possibility of alkoxymethylation of this group.

Alkoxymethylation of 2-aminothyazole was carried out by preliminary preparation of semiformal of corresponding alkanol. Paraform was used as a source of formaldehyde. Then a solution of an equimolar amount of 2aminothiazole in ethanol was added to a semiformal cooled to $5{ }^{0} \mathrm{C}$ :

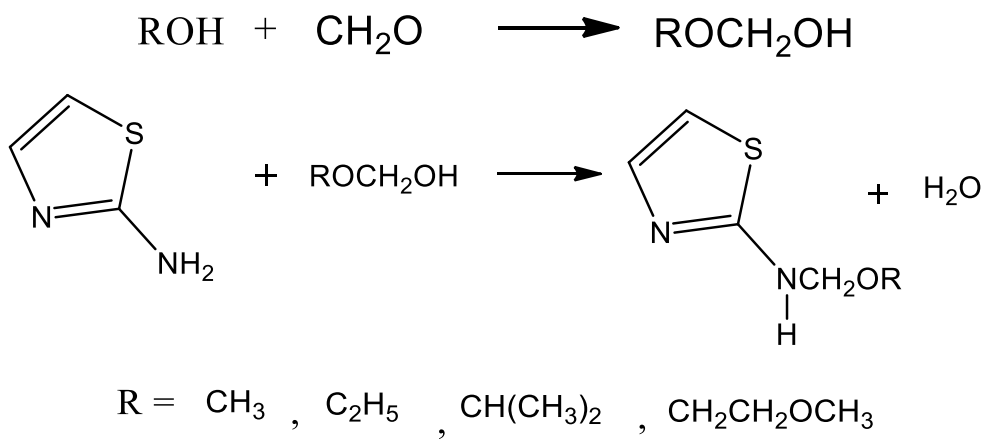

Synthesis of N-(ethoxymethyl)thiazol-2-amine

A three-necked reaction flask equipped with a mechanical stirrer, dropping funnel, thermometer, and reflux condenser was charged with $3.3 \mathrm{~g}(0.1 \mathrm{~mol})$ paraform, $4.6 \mathrm{~g}(0.1 \mathrm{~mol})$ ethanol and $1 / 2 \mathrm{KOH}$ granule. The reaction mixture was stirred at a temperature of $60^{\circ} \mathrm{C}$ until the paraform was completely depolymerized and semiformal formed, cooled to $5^{\circ} \mathrm{C}$, and a solution of $5.0 \mathrm{~g}(0.05 \mathrm{~mol})$ of 2aminothiazole in $40 \mathrm{ml}$ of ethanol was added dropwise. The mixture was stirred at $5^{\circ} \mathrm{C}$ for 2 hours and left at room tempetature overnight. Then $50 \mathrm{ml}$ of absolute benzene was added and the reaction water was removed azeotropically with benzene in a Dean-Stark trap. After cooling, the reaction mixture was filtered. Benzene was distilled off on a water jet. The resulting crystalline product was recrystallized from boiling ethanol. There was received $3.8 \mathrm{~g}$ of substance (yield $48 \%$ ). The rest of alkoxymethyl derivatives were prepared in a similar manner.

The obtained compounds are crystalline substances purified by recrystallization from boiling ethanol. Physio-chemical characteristics are given in Table 1 .

\section{Results and discussion}

The structure of the synthesized compounds was established by IR and ${ }^{\mathbf{1}} \mathrm{H}$ NMR spectroscopy data and their composition determined by element analysis. In the ${ }^{1} \mathrm{H}$ NMR spectra of these substances the following signals were observed: a broadened singlet 
which was characteristic for $\mathrm{NH}$ group in the range 2.47-2.86 m.d., and a singlet typical for $\mathrm{NCH}_{2} \mathrm{O}$ group in the range 5.20-5.25 m.d. Note that ${ }^{1} \mathrm{H}$ NMR spectroscopic data are given in Table 1.

In the IR spectrum of these compounds there was an absorption band in the region of $3183-3211 \mathrm{~cm}^{-1}$ typical for valence vibrations of $\mathrm{NH}$ group and there was no absorption band at
3400-3500 $\mathrm{cm}^{-1}$ which was typical for valence vibrations of $\mathrm{NH}_{2}$ group.

As shown by data of IR and ${ }^{1} \mathrm{H}$ NMR spectroscopy, the reaction of alkoxymethylation of 2-aminothiazole proceeds in the amino form with the formation of a monosubstituted $\mathrm{N}$ alkoxymethyl derivative.

As an example, the spectra of some obtained compounds are given in Fig. 1, 2, 3.

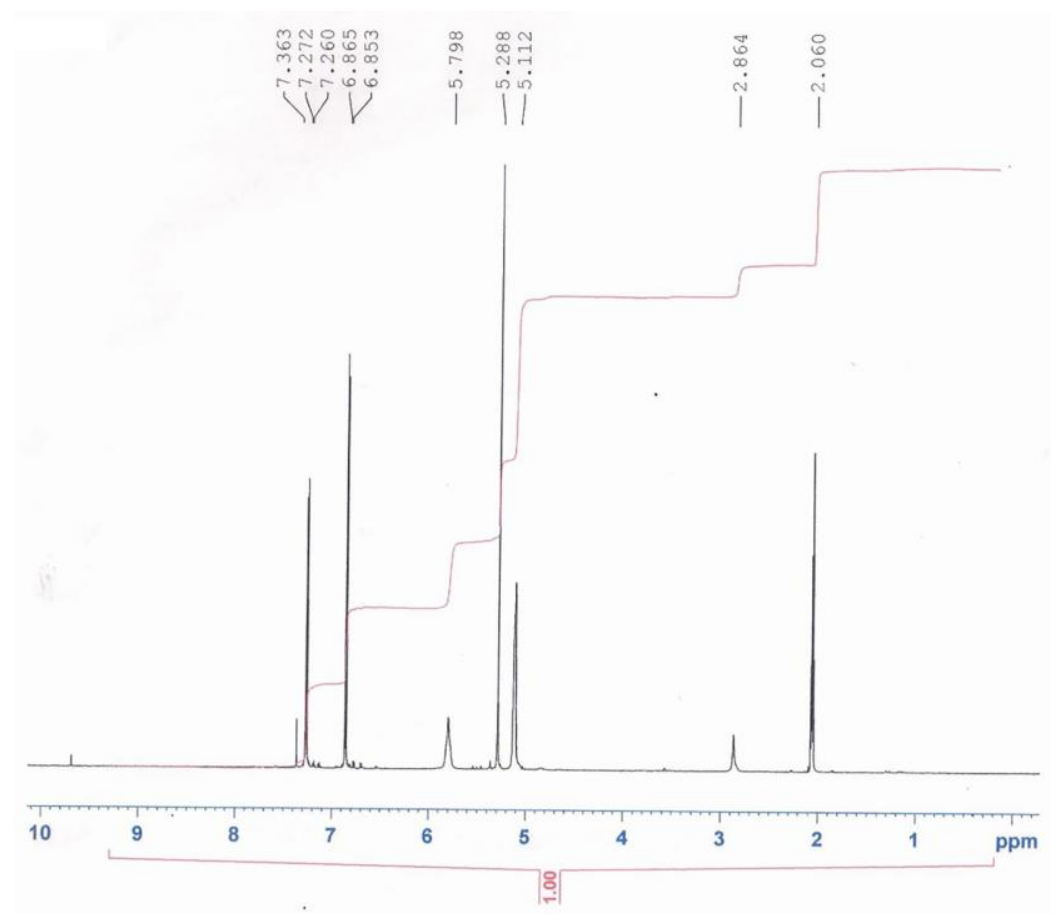

Fiq 1. ${ }^{1} \mathrm{H}$-NMR spectra of $\mathrm{N}$-(isopropoxymethyl)thiazol-2-amine

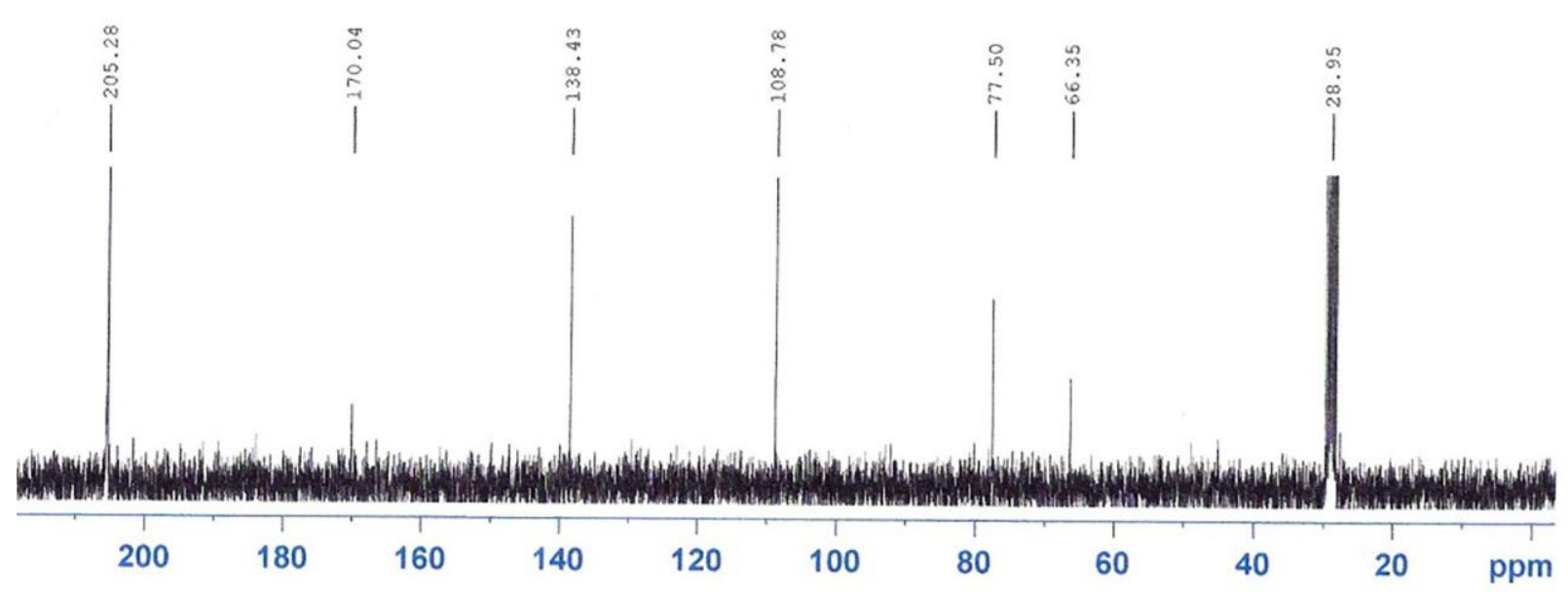

Fiq 2. ${ }^{13} \mathrm{C}$-NMR spectra of N-(isopropoxymethyl)thiazol-2-amine 


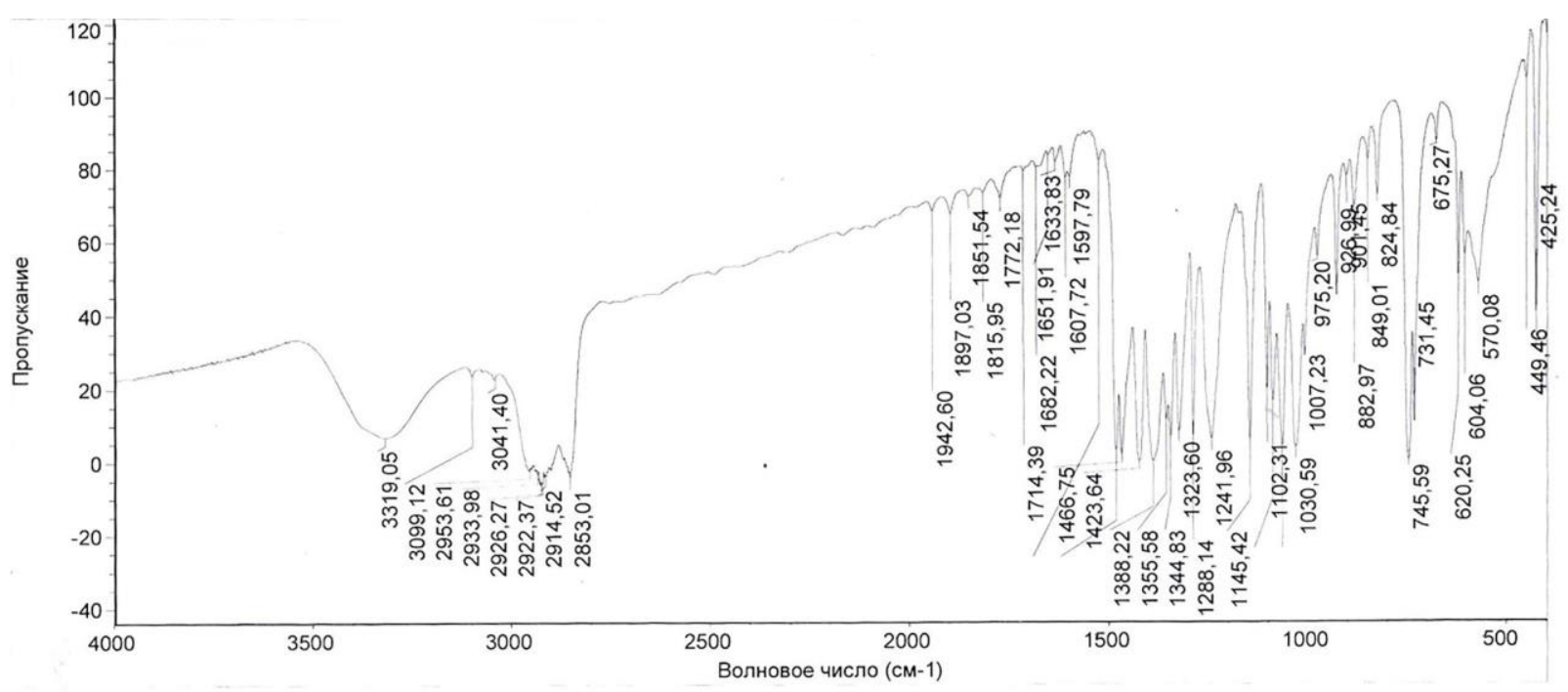

Fiq 3. IR Spectrum of N-(methoxymethyl) thiazol-2-amine

Table 1. Physio-chemical characteristics of $\mathrm{N}$-alkoxymethyl derivatives of 2-aminothiazole

\begin{tabular}{|c|c|c|c|c|c|c|}
\hline \multirow{3}{*}{$\begin{array}{l}\text { № } \\
\text { № }\end{array}$} & \multirow[t]{3}{*}{ Compound } & \multirow{3}{*}{$\begin{array}{l}\text { Melting } \\
\text { point, }{ }^{\circ} \mathrm{C}\end{array}$} & \multirow{3}{*}{$\begin{array}{c}\text { Yield, } \\
\%\end{array}$} & \multicolumn{2}{|c|}{ Content, $\%$} & \multirow[t]{3}{*}{${ }^{1} \mathrm{H}$ NMR spectrum, $\delta$, m.d. } \\
\hline & & & & $\begin{array}{c}\mathrm{N}, \\
\text { found }\end{array}$ & $\begin{array}{c}\mathrm{S}, \\
\text { found }\end{array}$ & \\
\hline & & & & \multicolumn{2}{|c|}{ calculated calculated } & \\
\hline 1 & $\begin{array}{l}\mathrm{NCH}_{2} \mathrm{OCH}_{3} \\
\mathrm{H}\end{array}$ & $126-127$ & 35 & $\frac{19.56}{19.44}$ & $\frac{22.61}{22.22}$ & $\begin{array}{l}3.24\left(\mathrm{~s} .3 \mathrm{H}, \mathrm{OCH}_{3}\right), 2.47-2.77 \\
\text { (broadened singlet } 1 \mathrm{H}, \mathrm{NH}), 5.20(\mathrm{~s} . \\
\left.2 \mathrm{H}, \mathrm{NCH}_{2} \mathrm{O}\right), 6.85-6.9(\text { d. } 1 \mathrm{H}, \mathrm{NCH}), \\
7.26-7.28(\text { d. } 1 \mathrm{H}, \mathrm{SCH})\end{array}$ \\
\hline 2 & $\begin{array}{l}\mathrm{NCH}_{2} \mathrm{OC}_{2} \mathrm{H}_{5} \\
I \\
\mathrm{H}\end{array}$ & $126-129$ & 48 & $\frac{18.01}{17.72}$ & $\frac{20.40}{20.25}$ & $\begin{array}{l}0.9-1.2\left(\mathrm{t} ., 3 \mathrm{H}, \mathrm{CH}_{3}\right), 2.4-2.8 \\
\text { (broadened singlet, } 1 \mathrm{H}, \mathrm{NH}), 5.05 \\
\left.\text { (broadened singlet, } 2 \mathrm{H}, \mathrm{OCH}_{2}\right), 5.25 \\
\left(\mathrm{~s} ., 2 \mathrm{H}, \mathrm{NCH}_{2} \mathrm{O}\right), 6.69(\mathrm{~d} ., 1 \mathrm{H}, \mathrm{NH}), \\
\text { 7.25-7.3 (d., } 1 \mathrm{H}, \mathrm{SCH})\end{array}$ \\
\hline 3 & $\begin{array}{l}\mathrm{NCH}_{2} \mathrm{OCH}\left(\mathrm{CH}_{3}\right)_{2} \\
\mathrm{H} \\
\mathrm{H}\end{array}$ & $120-121$ & 52 & $\frac{16.50}{16.37}$ & $\frac{18.9}{18.71}$ & $\begin{array}{l}\left.1.11-1.90 \text { (t., } 6 \mathrm{H}, 2 \mathrm{CH}_{3}\right), 2.37-2.82 \\
\text { (broadened singlet, } 1 \mathrm{H}, \mathrm{NH}), 5.10 \\
\text { (broadened singlet, } 1 \mathrm{H}, \mathrm{OCH}), 5.29 \\
\left(\mathrm{~s} ., 2 \mathrm{H}, \mathrm{NCH}_{2} \mathrm{O}\right), 6.68(\mathrm{~d} ., 1 \mathrm{H}, \\
\mathrm{NCH}), 7.23-7.27(\mathrm{~d} ., 1 \mathrm{H}, \mathrm{SCH})\end{array}$ \\
\hline 4 & $\begin{array}{l}\mathrm{NCH}_{2} \mathrm{OCH}_{2} \mathrm{CH}_{2} \mathrm{OCH}_{3} \\
\mathrm{H}\end{array}$ & $127-128$ & 30 & $\frac{16.41}{16.28}$ & $\frac{18.95}{18.60}$ & $\begin{array}{l}\left.3.19 \text { (s., } 3 \mathrm{H}, \mathrm{OCH}_{3}\right), 2.86(\mathrm{~s} ., 1 \mathrm{H}, \\
\mathrm{NH}), 5.11\left(\mathrm{t} ., 4 \mathrm{H}, \mathrm{OCH}_{2} \mathrm{CH}_{2} \mathrm{O}\right), 5.20 \\
\begin{array}{ll}\left.\text { (s., } \quad 2 \mathrm{H}, \quad \mathrm{NCH}_{2} \mathrm{O}\right), & 6,85-6,87 \\
\text { (broadened singlet. } 1 \mathrm{H}, & \mathrm{NCH}=), \\
7.261-7.272 & \text { (d., } 1 \mathrm{H}, \mathrm{SCH})\end{array}\end{array}$ \\
\hline
\end{tabular}


Table 2. Antimicrobial properties of $\mathrm{N}$-alkoxymethyl derivatives of 2-aminothiazole

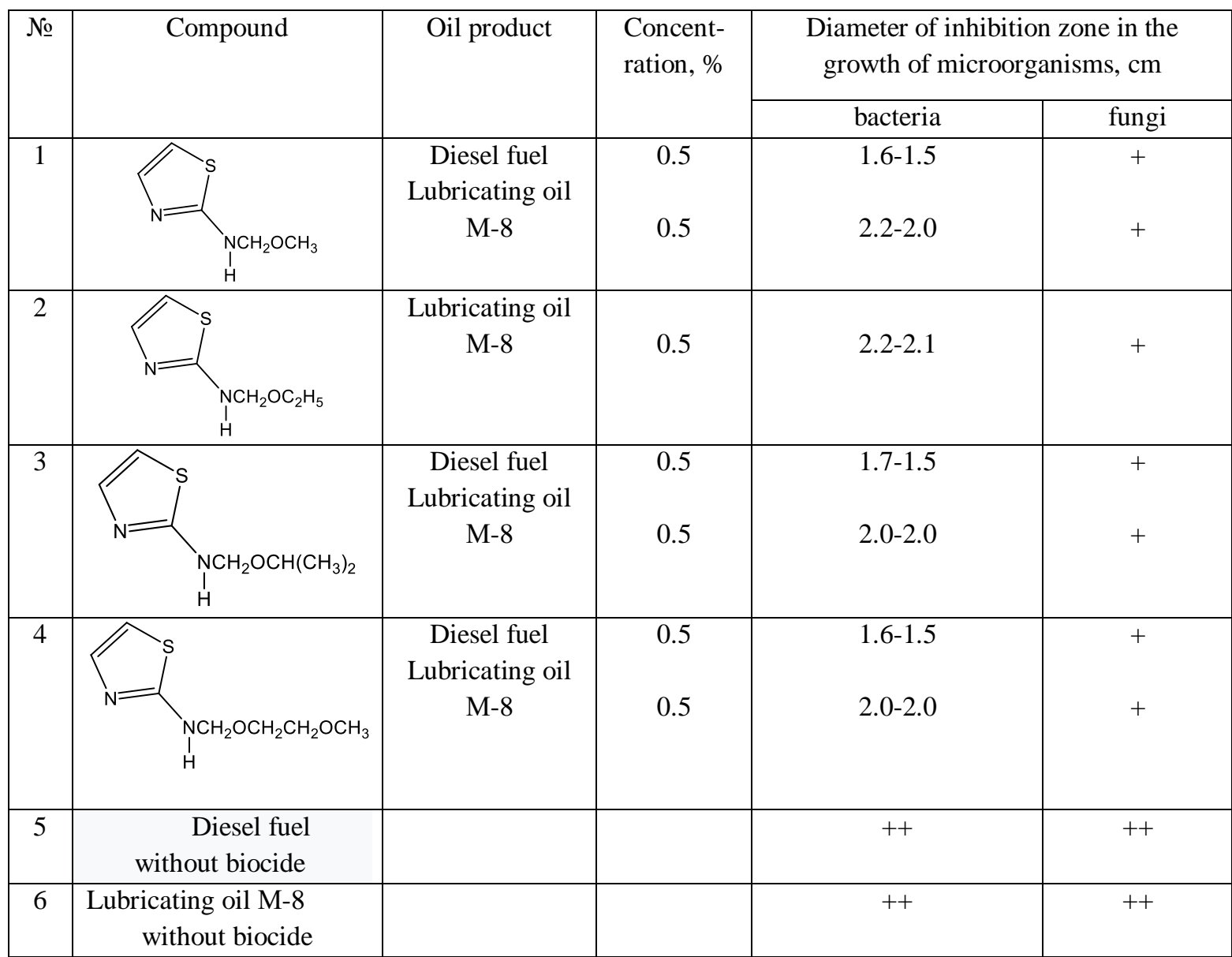

+ - growth of microorganisms.

Microbiological activity against microorganisms affecting oil products (lubricating oil M-8 and diesel fuel) was investigated of a unified system of protection against corrosion and aging in accordance with ГОСТ 9.082-77 "Oils and Lubricants. Methods of laboratory tests for resistance to bacteria", ГОСТ 9.052-88 "Oils and Lubricants. Methods of laboratory tests for resistance to mold fungi", ГОСТ 9.023-74 «Petroleum fuels. Method of laboratory tests for biostability of fuels protected by antimicrobial additives". Tests results are presented in Table 2.

Test results show that N-alkoxymethyl derivatives of 2-aminothiazole have good bactericidal properties. However, they are completely ineffective against mold fungi. Perhaps this is due to the poor solubility of these substances in petroleum products.
The presence of thiazole and alkoxymethylamine groups in the synthesized compounds suggests that they have potential anticorrosive properties. These properties were studied in respect of steel $\mathrm{CT}_{\mathrm{T}} \mathrm{3}$ according to ГОСТ 9.502-82 in an aggressive medium created by $0.1 \mathrm{~N}$ aqueous solutions of $\mathrm{HCl}$ and $\mathrm{H}_{2} \mathrm{SO}_{4}$.

$200 \mathrm{ml}$ of $0.1 \mathrm{~N}$ solution of acid was poured in a beaker, and the required amount of the test substance dissolved in $3 \mathrm{ml}$ of ethanol was added thereto. Two coupons (40x20x0.5) Ст-3 steel were immersed in this solution so that they did not touch each other. Three hours after, the metal coupons were rinsed under running water and dried. The thin plaque on them was cleaned with rubber, the coupons were rinsed with hexane, dried with acetone and weighed to the nearest $0.0001 \mathrm{~g}$.

Corrosion rate was determined by the formula:

$$
\rho=\frac{m_{1}-m_{n}}{s \cdot \tau}
$$


$\mathrm{m}_{1}$ - coupon weight before testing, $\mathrm{g}$.

$\mathrm{m}_{2}$ - coupon weight after testing, $\mathrm{g}$.

S- coupon area, $\mathrm{m}^{2}$.

$\tau$ - test time, hours.

Degree of corrosion protection was determined by the formula:

$$
Z=\frac{\rho_{0}-\rho}{\rho_{0}} \times 100 \%
$$

$\rho_{0}$ - corrosion rate without inhibitor, $\mathrm{g} / \mathrm{m}^{2} \cdot$ hour

$\rho$ - corrosion rate with inhibitor, $\mathrm{g} / \mathrm{m}^{2} \cdot$ hour

The test results are presented in Table 3.

Table 3. Test results of inhibiting properties

\begin{tabular}{|c|c|c|c|c|c|c|}
\hline \multirow[t]{2}{*}{ № } & \multirow[t]{2}{*}{ Inhibitor } & \multirow{2}{*}{$\begin{array}{l}\text { Concentra- } \\
\text { tion, } \mathrm{mg} / \mathrm{l}\end{array}$} & \multicolumn{2}{|c|}{$0.1 \mathrm{~N} \mathrm{HCl}$} & \multicolumn{2}{|c|}{$0.1 \mathrm{~N} \mathrm{H}_{2} \mathrm{SO}_{4}$} \\
\hline & & & $\begin{array}{l}\text { Corrosion } \\
\text { rate } \rho, \\
\mathrm{g} / \mathrm{m}^{2} \cdot \text { hour }\end{array}$ & $\begin{array}{l}\text { Protective } \\
\text { effect Z, \% }\end{array}$ & $\begin{array}{c}\text { Corrosion rate } \\
\rho, \\
\mathrm{g} / \mathrm{m}^{2} \cdot \text { hour }\end{array}$ & $\begin{array}{l}\text { Protective } \\
\text { effect Z, \% }\end{array}$ \\
\hline 1 & $\begin{array}{c}\text { Medium: } \\
0.1 \mathrm{~N} \text { acid solution } \\
\text { without inhibitor }+3 \mathrm{ml} \\
\text { EtOH }\end{array}$ & & 3.6 & & 5.4 & \\
\hline 2 & $\overbrace{\substack{\mathrm{NCH}_{2} \mathrm{OCH}_{3} \\
1 \\
\mathrm{H}}}^{\mathrm{S}}$ & 100 & 1.04 & 71 & & \\
\hline 3 & $\underset{\mathrm{H}}{\mathrm{N}} \mathrm{NH}_{2} \mathrm{OC}_{2} \mathrm{H}_{5}$ & $-\ll-$ & 0.40 & 88 & & \\
\hline 4 & 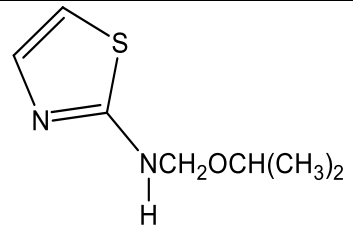 & $-\ll-$ & 0.35 & 90 & 0.41 & 92 \\
\hline 5 & & $-\ll-$ & 0.55 & 85 & & \\
\hline & $\begin{array}{l}\mathrm{NCH}_{2} \mathrm{OCH}_{2} \mathrm{CH}_{2} \mathrm{OCH} \\
1 \\
\mathrm{H}\end{array}$ & & & & & \\
\hline
\end{tabular}

Test results are indicative that synthesized 2alkoxymethyl derivatives of 2-aminothiazole possess anticorrosive properties. Their protective effect is $71-90 \%$ at a concentration of $100 \mathrm{mg} / \mathrm{l}$ in acidic medium created by $0.1 \mathrm{~N}$ solution of $\mathrm{HCl}$. The sample 4 has protective effect of $90 \%$ in $0.1 \mathrm{~N}$ solution of $\mathrm{HCl}$, and it exhibits practically the same protective effect of $92 \%$ in $0.1 \mathrm{~N}$ solution of $\mathrm{H}_{2} \mathrm{SO}_{4}$. 


\section{Conclusion}

Conditions of three-component condensation of 2-aminothiazole, paraform and aliphatic alcohols were worked out, and a number of 2-alkoxymethyl derivatives of 2aminothiazole synthesized. Their structure was proved out by the data of IR and NMR ${ }^{1} \mathrm{H}$ spectroscopy. It was established that synthesized compounds in the composition of petroleum products at a concentration of $0.5 \%$ exhibited high bactericidal properties. And they successfully protect ST-3 steel against acid corrosion caused by $0.1 \mathrm{~N}$ solutions of $\mathrm{HCl}$ and $\mathrm{H}_{2} \mathrm{SO}_{4}$.

\section{References}

1. Campbell M.M. Pyatichlennie geteritsikli/ Obshaya organicheskaya khimiya/ Kislorodsoderjashie, serosoderjashie i drugie geterotsikli. Pod. Red. P.G Sammes - Per. s angl./ Pod. Red. Kochetkova - M.: Khimia. 1985. Ch. 20.1 S. 442-558. [Comprehensive Organic Chemistry. The Synthesis and Reactions of Organic Compounds. Volume 4. Heterocyclic Compounds. Edited by P.G. Sammes. The City University. London. PERGAMON PRESS].

2. Adel A. Mohamed Arwa W. El-Harby. Substituted effect on the amino-imino tautomerizm of aminothiazoles. $J$. of molecular structure: Theochem. 2008, vol. 848, issue 1-3, pp. 52-61.

3. Uchikawa O., Fukatsu K., Suno M., Aono T., Doi T. In vivo biological activity of antioxydative aminothiazole derivatives. Chem. and Pharm. Bull. 1996, vol. 44, no. 11, pp. 2070-2070.

4. Manian A.K., Khase B.G., Kirtikar P.A., Sengupta S.R. Synthesis and study of 2amino-4-(substituted) benzoylthiazole derivatives as possible chemotherapeutic agents. Abstr. 42 ${ }^{\text {nd }}$ Indian Pharm. Congr., Minipal, 28-30 Dec., 1990. Indian J. Pharm. Sci. 1991, vol. 53, no. 3, p. 102.

5. Radchenko E.V., Payulin V.A., Zefirov N.S., Makhaeva G.F., Bolteva N.P., Serebrryakova O.G., Serkov I.V., Proshin A.N. Molekular Design of N,NDisubstituted 2-Aminothiazoline as selective carboxulesterase inhibitor. Russian Chemical Bulletin. 2016, vol. 65, no. 2, pp. 570-575.

6. Iram Batool et al. Synthesis, molecular docking and biological evaluation of new thiazolopyrimidine carboxylates as potential antidiabetic and antibacterial agents. Res. Chem. Intermediates. 2016, vol. 42 , no. 2 , pp.1139-1163.

7. Meissner, Anja; Boshoff, Helena I.; Vasan, Mahalakshmi; Duckworth, Benjamin P.; Barry, III Clifton E.; Aldrich, Courtney C. Structure-activity relationships of 2aminothiazoles effective against Mycobacterium tuberculosis. Bioorg. and Med. Chem. 2013. vol. 21, no. 21. pp. 6385-6397

8. Khalil, Ahmed; Edwards, Jessica A.; Rappleye, Chad A.; Tjarks, Werner. Design, synthesis, and biological evaluation of aminothiazole derivatives against the fungal

9. pathogens Histoplasma capsulatum and Cryptococcus neoformans. Bioorg. and Med. Chem. 2015, vol. 23, no. 3, pp. 532547.

10. Ramana M.M.V., Dubhashi Deepnandan S., D'Sousa Joseph. Synthesis of some new biologically active 5-Phenoxy-4-phenyl1,3-thiazol-2amines. J. Chem. Res. Synop. 1998, no. 9, p. 496.

11. Jain Renuka, Shipra. Synthesis, spectral studies and antimicrobial activity of some fluorin containing 2-amino-4-arylthiazoles. J. Indian Chem. Soc. 1997, vol. 74, no. 1, pp. 54-55.

12. Kelarev V.I., Grachova O.G., Silin M.A., Koshelev V.N., Golubeva I.A. Investigation of the action of amino and thio derivatives of sim-triazine as polyfunctional additives to lubricating oils. Neftepererabotka $i$ Neftekhimiya. 1997, no.12, pp. 29-32. (In Russian). 
13. Karpov K.A., Nazarenko A.N., Pikarevskii B.V., Potekhin V.M. Biocidal and anticorrosive effect of 2-aminothiazole derivatives to jet fuels. Russian Journal of Applied Chemistry. 2001, vol. 74, no. 6, pp. 998-1001.

\title{
2- AMINOTİAZOLUN N- ALKOKSIMETIL TÖRӘMӘLӘRİ VӘ ONLARIN MÜHAFIZZEDICİ XASSЭLӘRİ
}

\author{
V.M. Fərzəliyev, M.T. Abbasova, G.B. Babayeva, M.A. Mirzəyeva, Q.M. Quliyeva, L.R. \\ Səfərova, N.A. Oliyeva \\ AMEA Aşqarlar Kimyasi Institutu \\ AZ 1029, Bakı, Böyükşor şossesi, məhalla 2062. \\ e-mail:leyla.aki@mail.ru
}

\begin{abstract}
Alifatik spirtlərin formaldehidlə qarşılıqlı təsiri nəticəsində alınan poluformalların 2-aminotiazolla aminometilləşmə reaksiyası işlənib hazırlanmışdır. Alkosimetilləşmə əvvəlcədən müvafiq hidroksilsaxlayan birləşmələrin poluformalının alınması, sonra isə onun 2-aminotiazolun ekvimolyar miqdarı ilə təsiri nəticəsində aparılmışdır. Alınmış birləşmələrin quruluşu İQ- və NMR ${ }^{1} \mathrm{H}$ - spektroskopiya üsulu ilə təyin etmişdir. Müəyyən olunmuşdur ki, alkoksimetilləşmə ilkin 2aminotiazolun amino-formasında gedir və uyğun monoəvəzolunmuş 2-alkoksimetil törəməsinin alınması ilə nəticələnir. Mikrobioloji sınaqlar nəticəsində təyin edilmişdir ki, bu birləşmələr neft məhsullarını zədələyən mikroorqanizmlərə qarşı yüksək bakterisid xassəyə malikdirlər və $0.5 \%$ qatılıqda M-8 sürtkü yağı, eyni zamanda dizel yanacağını bakteriyalardan zədələnməyindən qoruyur, hətta onu məcburən zədələyəndə. Müəyyən edilmişdir ki, alınmış birləşmələr $100 \mathrm{mq} / \mathrm{l}$ qatılığında $\mathrm{CT}_{\mathrm{T}} 3$ poladı turş mühitdə korroziyadan qoruyurlar və N-(izopropoksimetil)tiazol-2amin ən yüksək qoruyucu effektə malikdir.
\end{abstract}

Açar sözlər: 2-aminotiazol, tautomeriya, amino-forma, imino-forma, alkoksimetilləşmə, poluformallar, alkoksimetil törəmələri, neft məhsulları, korroziya, mikroorqanizmlər, biozədələmə.

\section{N-АЛКОКСИМЕТИЛЬНЫЕ ПРОИЗВОДНЫЕ 2-АМИНОТИАЗОЛА И ИХ ЗАЩИТНЫЕ СВОЙСТВА}

\section{В.М. Фарзалиев, М.Т. Аббасова, Г.Б. Бабаева, М.А. Мирзоева, Г.М. Кулиева, Л.Р. Сафарова, Н.А. Алиева}

Институт химии присадок им акад. А.М. Кулиева Наџиональной АН Азербайджана AZ1029, Баку, Беюкшорское шоссе, кв.2062, e-mail: leyla.aki@mail.ru

Разработаны условия реакции алкоксиметилирования 2-аминотиазола полуформалями, получаемыми взаимодействием алифатических спиртов с формальдегидом. Алкоксиметилирование проводили путем предварительного получения полуформаля соответствующего гидроксилсодержащего соединения с последующим его взаимодействием с эквимолярным количеством 2-аминотиазола. Строение полученных соединений подтверждено методами ИК-, ЯМР ${ }^{1} \mathrm{H}$-спектроскопии. Установлено, что реакция алкоксиметилирования протекает в амино-форме исходного 2-аминотиазола с образованием соответствующего монозамещенного $\mathrm{N}$-алкоксиметильного производного. В результате 
микробиологических испытаний установлено, что эти соединения обладают высокими бактерицидными свойствами в отношении микроорганизмов, поражающих нефтепродукты и при концентрации 0.5 \% полностью защищают как смазочное масло М-8, так и дизельное топливо от поражения бактериями даже в условиях их принудительного заражения. Обнаружено, что полученные соединения при концентрации 100 мг/л обеспечивают защиту стали СТ-3 от кислотной коррозии, причем наибольшим защитным эффектом обладает $\mathrm{N}$ (изопропоксиметил)тиазол-2-амин.

Ключевые слова: 2-аминотиазол, таутомерия, амино-форма, имино-форма, алкоксиметилирование, полуформали, алкоксиметильные производные, антимикробная активность, антикоррозионные свойства, нефтепродукты, микроорганизмы, биоповреждение. 